\title{
An Integrated Fundus Image Segmentation Algorithm for Multiple Eye Ailments
}

\author{
Parul Datta $^{1}$, Prasenjit Das ${ }^{2}$ and Abhishek Kumar ${ }^{3}$ \\ ${ }^{1,3}$ CUSET, ${ }^{2}$ CUSCA, Chitkara University, Himachal Pradesh, India \\ *Corresponding Author: Parul Datta; Email: parul.datta@chitkarauniversity.edu.in
}

\begin{abstract}
The detection of eye illnesses requires a thorough inspection of all of the eye's structures. Most significantly, the presence of blood vessels, an optical disc, and any other unwelcome objects, if any are discovered, is critical in determining the type of eye disease present. Specifically, the goal of this research is to establish a thorough segmentation framework that will aid in the detection of anatomical anomalies in the eye. A novel segmentation technique for analyzing blood vessels, optical disc health, and the presence of exudates has been added into the software. - It was decided to add the detection of aberrant objects such as exudates into the algorithm in order to develop a generic segmentation method. In order to verify the accuracy of the segmentation at each stage, random sampling is employed at each stage. The segmentation is then validated using the intersection over union metric. The accuracy of the integrated segmentation method as a whole is 91.66 percent.
\end{abstract}

Keywords: Segmentation; fundus images; convolution filters; diabetic retinopathy; Otsu

\section{ARTICLE INFORMATION}

Author(s): Parul Datta, Prasenjit Das and Abhishek Kumar

Received: Dec 01, 2021; Accepted: Feb 01, 2022; Publis hed: Feb 10, 2022 e-ISSN: 2347-470X;

Paper Id: IJEER120115;

Citation: doi.org/10.37391/IJEER.090406

Webpage-link:

https://ijeer. forexjournal.co.in/archive/volume-9/ijeer-090406.html

\section{INTRODUCTION}

One of the most critical challenges for those practicing ophthalmology in the COVID-19 era is reducing the danger of COVID-19 cross-infection while also implementing effective infection control procedures in their practices. It is critical to clean "high-touch" surfaces on a regular basis and to keep a high level of sanitization on those surfaces. In outpatient treatment, a thorough dual screening and triaging approach is required in order to identify patients who are at high risk of infection and to ensure that they are properly segregated from the rest of the patient population. To limit communication between inpatient and outpatient treatment facilities to a minimum, it is vital to maintain the inpatient and outpatient treatment facilities completely distinct. To minimize patient attendance rates, the most effective option for the time being is to put in place administrative measures to make them lower. There has been an increase in the need for frequent modifications and rescheduling of appointments. Remote technology, despite its rising popularity in the investigative field, cannot fully substitute for the need to conduct investigations in person when doing so is absolutely necessary in many situations. The latest advances in mathematics and machine learning, according to studies published in high-impact journals in the fields of ophthalmology and computer science, are being used by many researchers, and indeed by many corporations, to improve the workflow associated with the clinical diagnosis and treatment of eye diseases. However, due to the fact that multi-disease models and applications are still in the early stages of development, some improvisation is required. Researchers are increasingly concentrating their efforts on highly specialized elements of eye issues and diseases, according to an exploratory review of the most recent study literature. One area of concentration for some researchers is developing systems that can identify morphological changes that occur in the blood vessels of the eyes as a result of diabetes, while another area of focus for other researchers is the health analysis of the optical discs of the eyes, among other things. The screening of individuals in a short period of time is therefore not achievable due to limited or single eye disease-based technology setups. The current extreme circumstances necessitate the development of technology that can reduce the frequency of patient visits while also enabling no-touch environments. Construction of technologies that scan human subjects for many diseases in a single visit while under the supervision of a medical practitioner can accomplish this. Blood vessels are organs of the body that carry body fluids in different parts of the body [3]. The medical world recognizes four kinds of blood vessels: arteries, veins, capillaries and sinusoids. Each type of blood vessel has a different function and fluid to carry from one destination to another. The tubes that carry blood from the heart to the other body parts are called arteries. These arteries may have branches known as arterioles. The tubes that bring back the blood fluid from various parts of the body to the heart are veins. Veins carry impure blood, and their branches are also known as veins. By monitoring the morphology, size, volume of the arteries and veins of the eye organ, characterization of eye disease can be done. These observations are typically done by a medical practitioner [4]. The medical specialist examines the various parts of the eye to diagnose eye diseases that include eyeball, retina, cornea, veins, arteries, and various other parts of the eyes $[5,6]$. If there is some abnormality in different parts of the eyes and inference is drawn in conjunction with other clinical findings, particular eye disease is detected. The manual process of detecting eye disease is highly specialized and tedious work, and at the same time, it is prone to different kinds of human bias 
and errors. Many researchers, as mentioned earlier, are employing multiple stack technologies to develop detection of eye problems to overcome this challenge and to leverage the latest technology [7]. For a computer-based system to successfully detect eye diseases requires the collection of existing case studies that cover multiple range of modalities is the first requirement [7]. For this, many researchers, institutions and organizations have initiated curating images related to eye diseases and publicly have made them available. With the help of publically available resources, the existing ecosystem of eye detection technologies can be improved further so that inadequacy of the limited capacity in terms of the number of diseases it can detect can be increased $[8,9]$.

Further, this research document has multiple sections and subtopics for maintaining clarity and readability as per the logical sequence. Section 2 includes the literature work related to the paper. Section 3 discusses the material and methods. Performance Analysis of the segmentation process with respect to different parts of the eyes is done in Section 4. The last two sections, Section 5 and Section 6 are dedicated to discussion and recommendation for future directions in context of this research, respectively.

\section{LITERATURE REVIEW}

This section discusses two topics. The first topic discusses the role of image processing and the second part discusses the general framework of detection and classification that are currently in practice for detecting eye diseases.

\subsection{Role of Image Processing}

It can be understood from the current literature survey that multiple samples of image modalities are managed for identifying eye disorders $[10,11]$. The most frequently used modality is the use of fundus images. The fundus image technology provides a convenient way of analyzing abnormalities in the eyes. Typically, when there is some ailment in an eye: either there are red spots [12], yellow circles [13], white spots, or cotton type blurry objects in the eye [14]. There might be a change in the size of the optical disc [15], a change in the number and dimensions of veins/arteries in the eyes, or simply an abnormal variation of texture in the retina area of the cornea area [15]. Hence, the objective of segmentation algorithms is to extract and segment those areas that give a clear-cut indication of a problem in the eye and clinical evidence that there is a specific type of eye disease. For this, researchers are using image processing techniques to remove the technical issues that would impact the accuracy of detection of eye disease.

The standard deviation values computed by medical specialists for each eye segment can be utilized to detect any abnormal changes in the eye. By segmenting the blood arteries separately, a computer algorithm aided by machines can assist clinicians in diagnosing and quantifying the disease's effect on the eyes. By segmenting different parts of the image, more precise pathology can be done and it will assure image based evidence care for ocular and medical disorders. The form, thickness, and texture of the eye regions aid in the analysis of the overall eye health [15-18]. If the shape, colour, and texture of the various parts of the eye (arteries, optical disc, eyeball size, and veins) deviate from the normal pattern of growth, the medical professional will be able to diagnose an eye condition [19]. There are a limited number of works that work on multiple eye parts for detection of multiple ailments. Typically, the focus of the contemporary papers is work on specific parts such as blood arteries.

Technically, it is known that noise enters the retina fundus images in many cases, which needs to be removed. For this, researchers either normalize the images or use methods that would remove the non-uniform illumination [20, 21]. Researchers have given empirical evidence that the green channel (RGB colour model) is the most appropriate for extracting red and yellow spots of the eyes [22]. The extraction is done by improving the contrast with the help of methods such as contrast stretching, adaptive contrast enhancement algorithms [23], and some have even used fuzzy logic to improve the contrast. By improving the contrast, the difference between the different intensities is increased, and for the segmentation algorithm, it becomes easy to segment that area. Many authors have demonstrated in their work that the variation in the background can be reduced by image smoothing filters and histogram equalization methods [20]. Researchers are also using shade correction methods for reducing non-uniform illumination areas in the eyes [24]. This way, the image is transformed for a better outcome in terms of segmentation. Many researchers have specifically targeted different parts of the eyes that get affected due to a particular kind of eye disease [7].

\subsection{General Segmentation Frameworks}

The detection frameworks of eye diseases can be understood at two levels. Lesions are analyzed in the first level, followed by image analysis to detect eye disease in the second level. In the case of the lesion approach, location, position and frequency are analyzed to determine the pattern of lesion development. From the lesion patterns, various kinds of inferences, such as the degree of severity of the disease, are taken in conjunction with clinical studies for detecting eye disease. The image-based detection evaluates eye disease signatures using multiple functions to identify a potential area in the eye anatomy for signs of abnormalities. This essentially includes detection of lesions medically referred to as micro aneurysms [25], hemorrhages [26], and exudates (soft or hard) [25]. The challenge in image processing is that it necessitates first addressing identifying and eliminating similar objects. Objects have similar geometric and texture features, e.g. red spots and blood vessels. Hence, on investigations, it can be found that researchers adopt various approaches in segmenting the region of interest for detecting eye ailments from fundus images. Most common methods of segmentation include the use of region merging methods, edge detection filters, pixel clustering algorithms such as k-means, local or global thresholding algorithms such as Otsu, ISO data and many more [27, 28, 29, 
30, 31]. The current citations in this context show that the focus of researchers was to construct $\mathrm{CNN}$-based algorithms for segmenting the blood vessels [32]. It can be observed that the use of UNet is most common in this context. It should also be noted that Convolutional Neural Network (CNN) based approaches require much augmentation in terms of data size, data augmentation, selection of convolution filters, selection of hyper-parameters for optimizing the $\mathrm{CNN}$ performance, and it has been found that in many cases, building segmentation models is a time-consuming process. Hence, in this research work, the attempt was to build a segmentation system that works with combined approaches or hybridization methods for streamlining the segmentation process. This paper demonstrates a generic algorithm that can quantify multiple eye problems, including detection of exudates and analysis of blood arteries and optical disc. According to experts, different eye diseases have different detection and identification problems. In many cases, the shape and geometry of the optical disc of the eyes give a clear indication of eye disease [33]. In detecting Diabetic Retinopathy (DR), a deep and thorough examination of blood vessels is essential. In the case of cataracts and glaucoma, analysis of exudates is critical. Therefore, many characteristics of DR, such as micro aneurysms, hemorrhages, and exudates, must be covered for a reliable detection solution. Hence, for constructing a standard eye problem detection algorithm, segmentation of optical disc, exudates, and blood vessels are essential.

In conjunction to funduscopic, fluorescein angiography (FA), and optical coherence tomography (OCT), other methods for measuring retinal blood flow dynamics, such as optical coherence tomography angiography (OCTA), and laser speckle flowgraphy, are being developed (LSFG) by current scientific community for the advancement in ophthalmology technologies. Image analysis is the first and most important step in the diagnosis process in all of these technologies. When following the logical workflow of image analysis, the initial step is picture segmentation. In order to establish prototypes in an ophthalmic image analysis pipeline that can be used across imaging modalities, it is clear that segmentation and quantification must be developed. This paper describes how to do so. The paper explores the use of previous segmentation methods to improve better workflow to justify foundations of image segmentation for multi-disease systems that can be made in future.

The Digital Retinal Images for Vessel Extraction (DRIVE) dataset [21] has been designed for the development and validation of retinal segmentation algorithms and not for DR detection and does not cover the full spectrum of DR severity, camera variations, and patient demographics. Hence, data fusion and data augmentation should be considered to overcome the inadequacies. Therefore, the problem revolves around constructing a combination segmentation algorithm for detecting multiple eye diseases and covering the problem of low quality of fundus images due to lens opacifications and interoperator variability in image quality.

\section{MATERIAL AND METHODS}

This section details the techniques required to overcome the obstacles associated with segmenting various components of the eye, such as blood veins, optical discs, and so on. The workflow for the segmentation procedure is readily apparent in Figure 1. There are three primary sources of data for this research: DRIVE [21, 29], IDRiD [34] and SUSTech-SYSU dataset. By previous researchers the DRIVE database was planned and developed to facilitate comparative studies on blood vessel segmentation in human retinal representations. Segmentation and visualization of retinal blood vessels and their morphological characteristics, such as the roundness of the optical disc, vessel bifurcation patterns, and matter orientations, are used to screen and diagnose. Diagnosis, screening and treatment of various cardiovascular and ophthalmologic illnesses such as heart attack, diabetes, hypertension etc. use segmentation of the retinal blood vessels. This further helps to establish the definition of their morphological characteristics such as length, width, tortuosity, and orientation.

Technical analysis of the blood vessels located in the eyes benefits in investigations of other screening programs and diagnoses protocols. The analysis helps to find clinical correlations and association between factors that are causing a particular medical eye condition in the patients. Mathematically methods and algorithms help to establish a basis between the influencing factors such as vessel thickness, shape with respect to ailments. Additionally, these screening programs will aid in the progress and research of laser surgery with computer assistance. The second dataset pertains to the demographic region of India. The IDRiD data collection contains 516 images of clinical quality obtained from an eye check-up facility in India. This clinical corpus is composed of patients with diabetic retinopathy and diabetic macular edema (DME).

The SUSTech-SYSU dataset is the third dataset [35]. This dataset was created for the aim of detecting exudates and gradation. Micro aneurysms, hard exudates, soft exudates, bleeding, microvascular abnormalities, intraretinal, and neovascularization are all examples of DR lesions. According to recent research, the size and amount of hard exudates may have serve as a potential discriminating predictor of the severity of DR. Increased production of hard exudates is a sign of a serious underlying ailment that requires immediate medical attention. Multiple databases were aggregated to aid the construction of the algorithms that can detect DR, DME, cataract, glaucoma, and healthy eye persons. The process was referred to as data fusion. Data fusion algorithms leverage multiple disease data sets for constructing image processing functions that work on heterogeneous disjoint sets that can support multi-disease detection systems. Some authors refer to such procedures as data adaptation also. Data adaptation is a process by which a data set is constructed to help the learning component of the detection system to discriminate between the various disease modalities and deliver an effective solution. Data augmentation, data fusion, and data adaptation immensely help overcome building generic systems of detection. 


\subsection{Generic Segmentation Algorithm}

This section explains the steps taken for segmenting each part of the eye that can provide hints about the kind of abnormality in the eye. Normality test defines the normal standard deviations of thickness and sizes of various eye parts. This test also defines the normally expected colour, texture and shape of the eye parts. Suppose there is some degree of deviation from these values, then there is a fair symptom that a large population of the world is suffering from some kind of eye ailment. Many remain undiagnosed until it becomes late, secondly lack of facilities forces us to rethink about systems of eye problem detection. Hence, an automatic algorithm that can extract all the parts of the eyes and can help in the qualitative and physiological analysis of the eye in terms of its health requires steps that include: extraction of eyes' blood vessels, extraction of optical disk boundary, and extraction of soft and hard exudates in the eye.

\subsubsection{Segmentation of Blood Vessels}

The resource input to the segmentation process includes the eye fundus images, gradient filters, global thresholding algorithms functions and constant threshold values.
The recognition of blood vessels was contingent on our ability to work out the filters of the numerous segments that comprised the whole image accurately. The rate, at which intensity of pixel changed, enabled the detection of object borders and edges in images. The gradient became more visible after changing the image colour model to CIELAB colour space. However, as a result of undesirable artefacts and noise, the image derivative rapidly changed, resulting in the detection of incorrect edges or segments of images. As a result, following pre-processing processes such as de-noising was done. This was accomplished through the use of two dimensional image convolutional filters. The information in Table 1 pertains to the filter qualities utilized in this study. The complete set of convolution kernels is based on the images constructed using derivative computations that make filtered images. This way it aids in detecting areas that have changed in the image semantics.

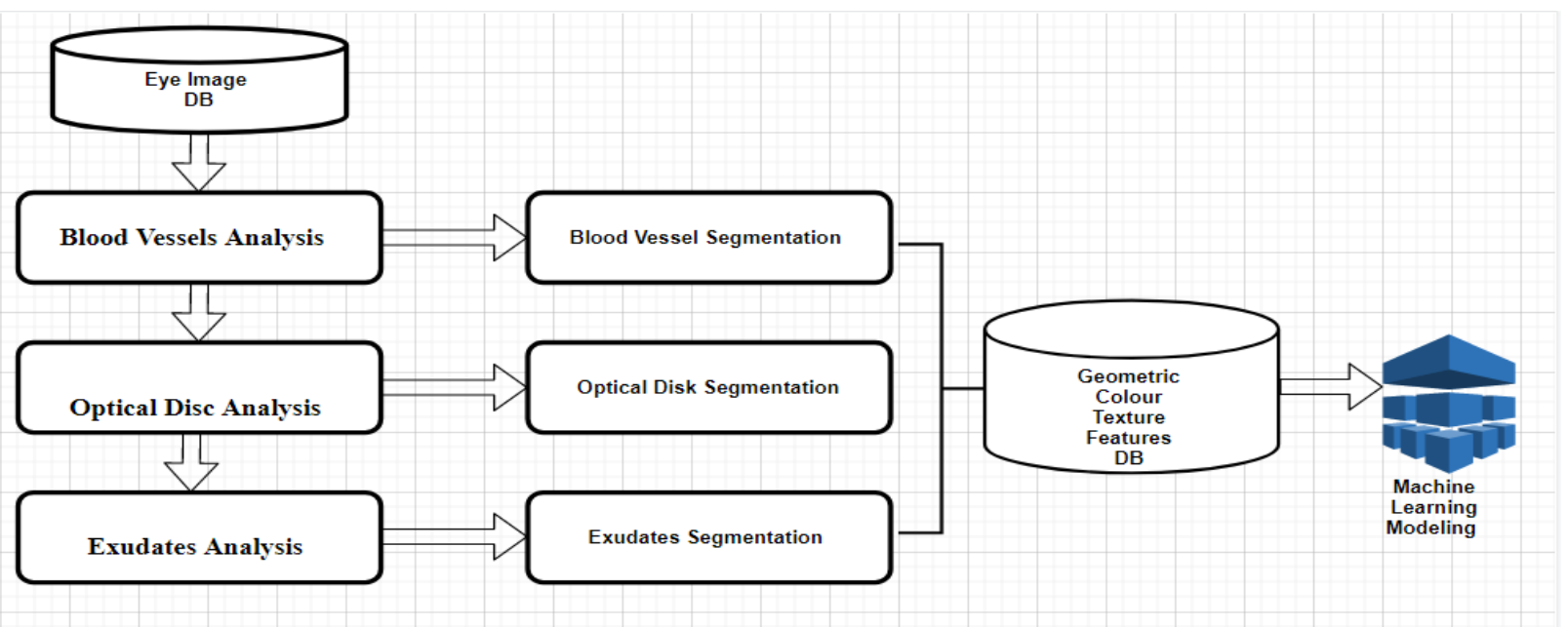

Figure 1: Logical workflow of segmentation process

Table 1: Convolutional Filter Attributes

\begin{tabular}{|c|c|c|c|c|}
\hline Sr. No. & Convolutional Filters & Size & Attributes of filters & Details of filters \\
\hline 1 & Prewitt & $3 \times 3$ & 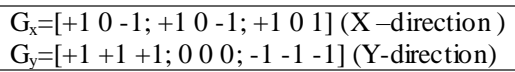 & Horizontal edge emphasizing filter \\
\hline 2 & $\begin{array}{l}\text { Laplacian of Gaussian } \\
\text { (LOG) }\end{array}$ & $5 \times 5$ & $\begin{array}{llllll}{\left[\begin{array}{cccccc}0 & 0 & -1 & 0 & 0 \\
0 & 1 & -2 & 1 & 0 \\
1 & 2 & -16 & 2 & 1 \\
0 & 1 & 2 & 1 & 0 \\
0 & 0 & 1 & 0 & 0\end{array}\right]}\end{array}$ & $\begin{array}{l}\text { Since, the derivatives of image are receptive } \\
\text { to the noise. Smoothening of image is done } \\
\text { by using filter such as Gaussian }\end{array}$ \\
\hline 3 & Laplacian Filter & $3 \times 3$ & $\left.\begin{array}{lll}{[0} & 1 & 0 \\
1 & -4 & 1 \\
0 & 1 & 0\end{array}\right]$ & Approximation of the 2D Laplacian filter \\
\hline 4 & Low Pass Gaussian Filter & $3 \times 3$ & Value of sigma is 0.1 & Gaussian low pass \\
\hline 5 & Sobel & $3 \times 3$ & 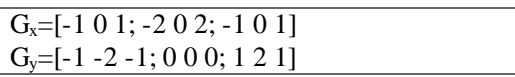 & Horizontal edge emphasizing filter \\
\hline 6 & Mean & $9 \times 9$ & $1 /($ kernel size $)$ & Mean intensity of the image is the basis \\
\hline
\end{tabular}


The following stage is to develop the segmentation algorithm in such a way that the picture processing does not require excessive computing and storage assets. To extract the coefficients from each image, the mathematics of Principal Component Analysis (PCA) was used. However, prior to this stage, the image matrix's colour model was modified to create a more accurate approximation of pixel intensities for segmentation, which transform image into invariant structure and at the same time decreases the digital footprint in terms of matrix size.

PCA is done using the following steps:

1. Calculate the 'Ii' image matrix's covariance.

2. Sort out the calculated Eigen vectors and their related values.

3 . To generate a new matrix with ' $k$ ' dimensions, select the first ' $k$ ' eigenvalue. This phase assists in determining the primary directions of difference in the image matrix.

4. Transmute the original image in dimension into new ' $\mathrm{k}$ ' dimensions

As briefly discussed above, steps 4 and 5 involve contrast enhancement and filter application.

Global thresholding techniques were used in this step to aggregate pixels with intensity larger than the level or threshold determined by the automatic thresholding method.

Additionally, pixels that were too tiny to be called blood veins were removed at this step. ISOdata and Otsu were compared. The Otsu algorithm uses the concept of clustering is based on the concept of minimizing variation between classes. ISOdata's pixel clustering algorithm is based on separating and merging groups according to their standard deviation.

Table 1 summarizes the outcomes of these approaches for various combinations of convolutional filters.

Background pixels were removed (Filtered picture 'JFi' obtained using the filters listed in Table 1) to get intensity values that corresponded to the blood vessels. This was accomplished through the use of set theory and the subtraction method:

$$
\mathrm{I}_{\mathrm{i}}=\mathrm{J}_{\mathrm{Fi}^{-}} \mathrm{J}_{\mathrm{ii}}
$$

Where ' $\mathrm{J}_{\mathrm{Fi}}$ ' is the filtered matrix of the image that contains pixels of the blood vessels and ' $\mathrm{J}_{\mathrm{ii}}$ ' is the optimized (stretched) contrast matrix of the image.

Finally, the resulting image was applied to the complement mathematical process to get the concluding segmented image.

\subsubsection{Blood vessel segmentation output analysis}

Manual visual observations demonstrate that segmentation output by Otsu (global thresholding) in combination with the mean filter is the best of all studied combinations. The blood vessels' shapes were crystal clear. The LOG, Prewitt and Laplacian operations do not segment effectively in achieving the precise Region of Interest (ROI), as seen in the following eye images.

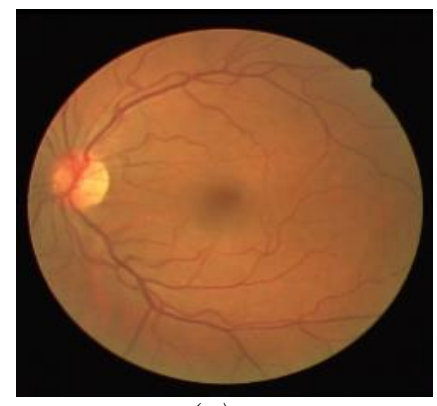

(a)

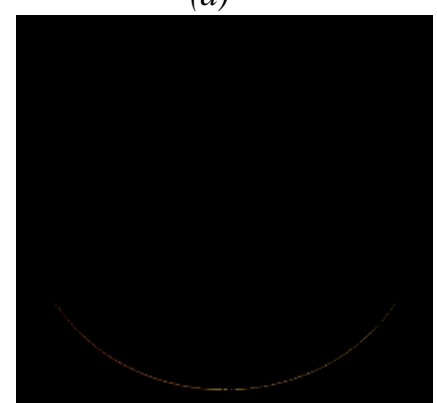

(c)

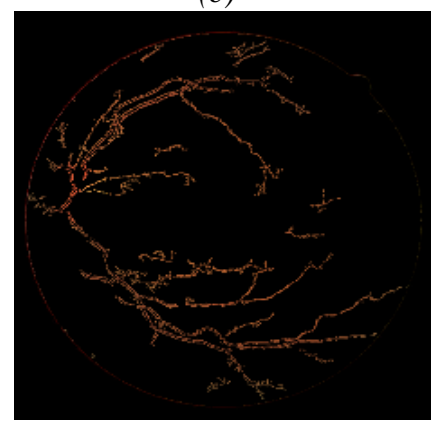

(e)

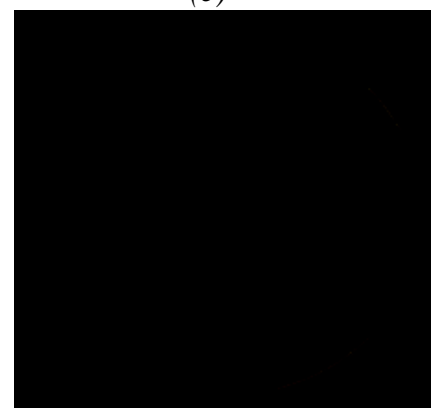

$(g)$

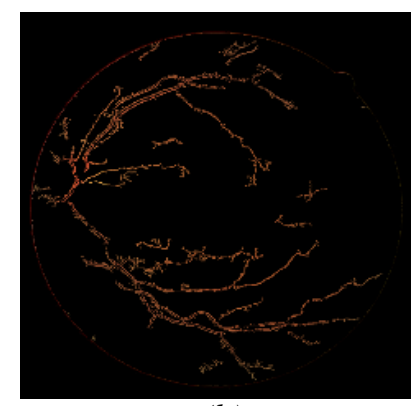

$(b)$

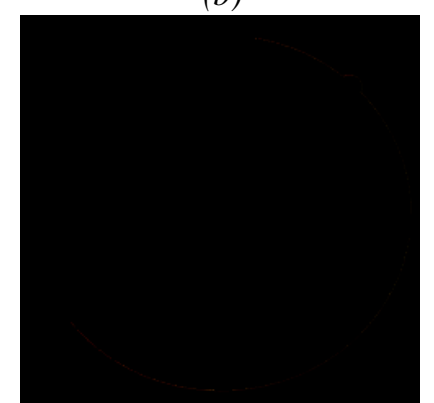

(d)

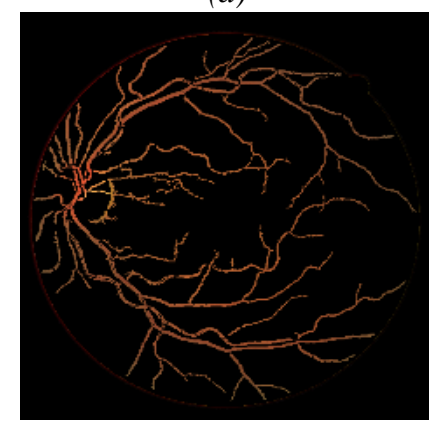

$(f)$

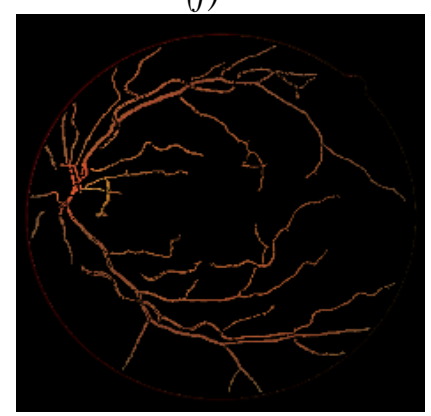

(h)
Figure 2: Segmentation results from left angle (a) Actual image (b) Sobel (c) Prewitt (d) Laplacian of Gaussian (e) Gaussian (f) ISOdata and Mean (g) Laplacian (h) Otsu and Mean

The outcome of the segmentation algorithms is shown in Figure 2. It demonstrates that blood vessels were conspicuous and appropriately segmented. It appears as though the Laplacian, LOG, and Sobel filters, in conjunction with Otsu and ISOdata, offer inadequate indications for blood vessel extraction. As a result of the sobel operator's ability to smooth the image data, the Otsu and ISOdata algorithms failed to group the pixels. 


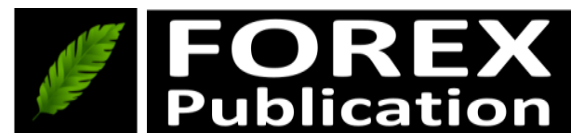

Open Access | Rapid and quality publishing
Segmentation failed completely in the case of Laplacian and LOG filters. In this sample, the poor segmentation outcome can be due to the fact that the second-order derivative of the space was not able to distinguish regions with sudden gradient changes in the image. As illustrated in Figure 2, when used in conjunction with Otsu, an independent implementation of Gaussian smoothing produced superior output. The mean filter output, when combined with ISOdata or Otsu, resulted in highquality segmentation. The mean filter looks to be more effective in reducing noise and smoothing the image than the other filters. As a result of this, the image was generated with a higher level of segmentation result.

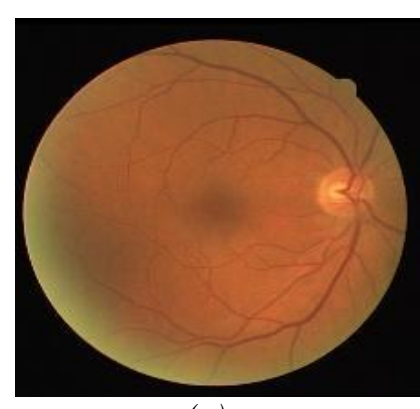

(a)

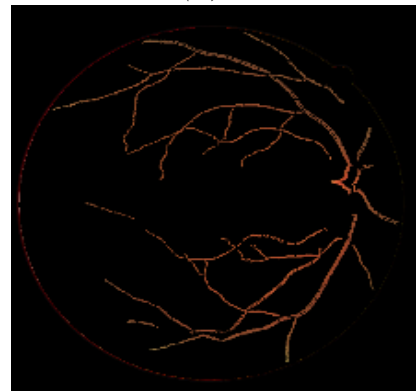

(c)

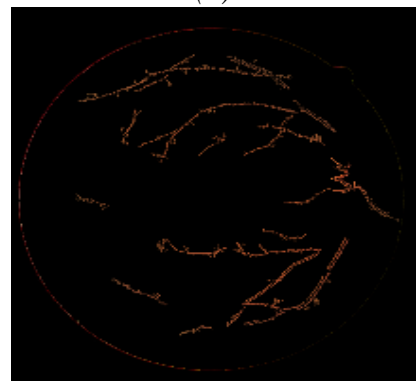

(e)

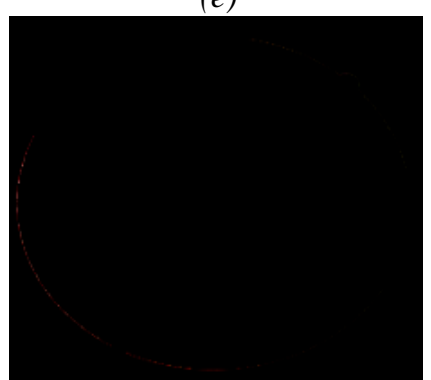

(g)

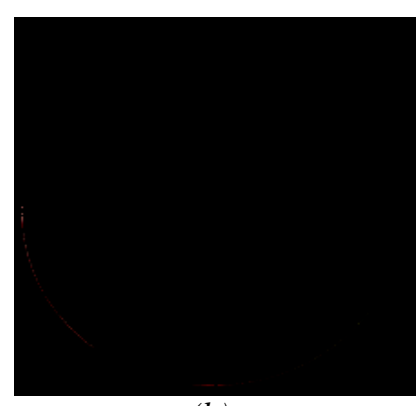

(b)

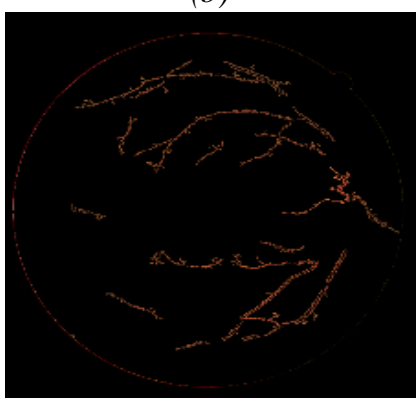

(d)

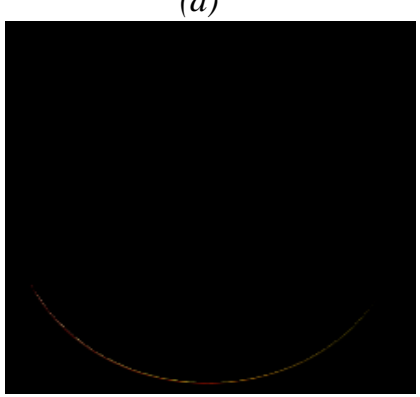

$(f)$

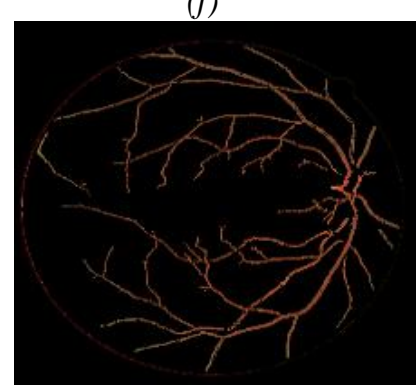

(h)
Figure 3: Segmentation results from right angle (a) Actual Image (b) Laplacian (c) Otsu and Mean (d) Sobel (e) Gaussian (f) Prewitt (g) Laplacian of Gaussian (h) Mean and ISOdata
The outputs of all the segmentation techniques employed to images with the different angles are depicted in Figure 3. These findings were consistent with those obtained with left-angle pictures, as illustrated in Figure 2. The mean filter appeared to be superior in its ability to manage certain types of noise.

In these images, there were a few uncommon pixel values that were distinctly unrepresentative. Additionally, the mean filter eliminated the need for edge interpolation. If this occurred, the margins would have blurred, resulting in the appearance of hazy blood vessels.

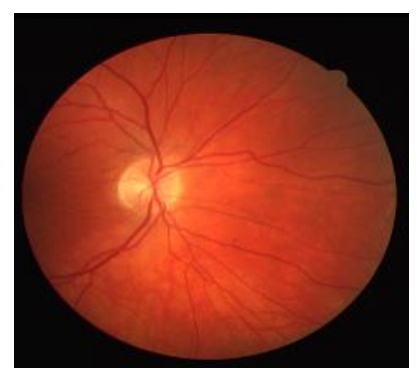

(a)

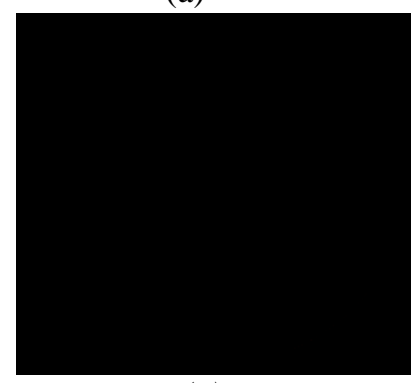

(c)

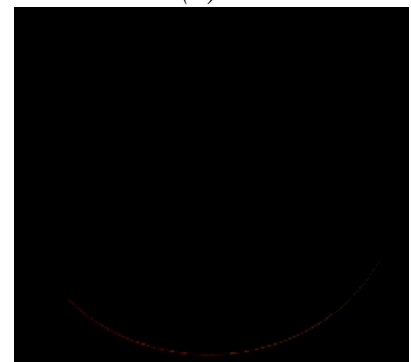

(e)

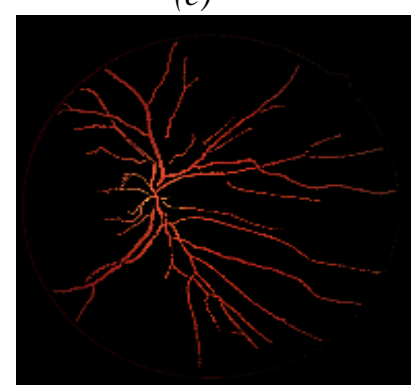

$(g)$

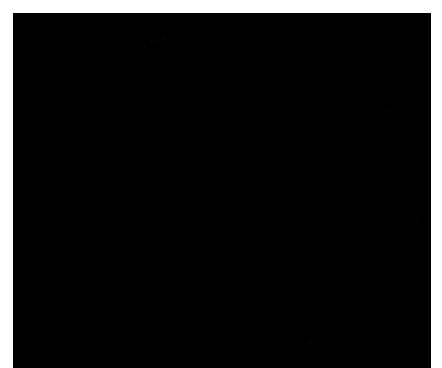

(b)

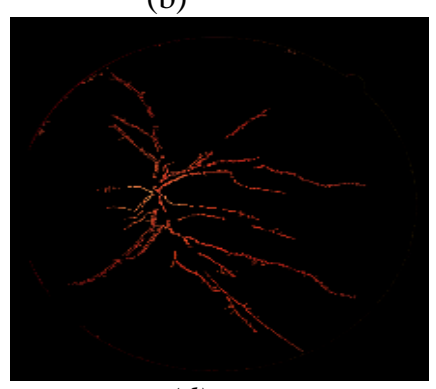

(d)

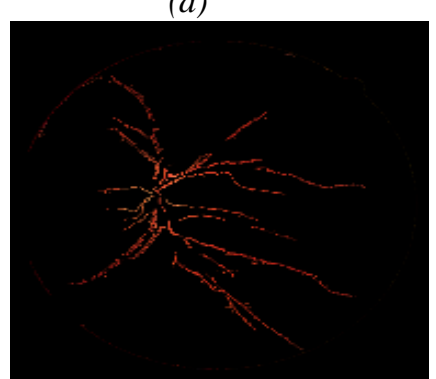

$(f)$

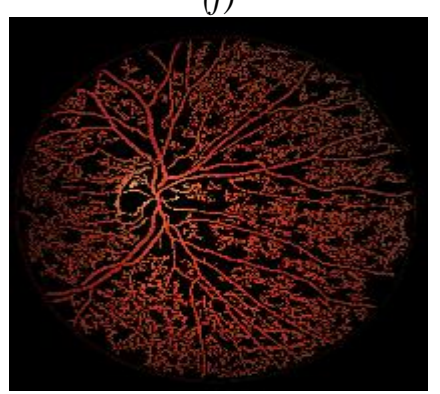

(h)
Figure 4: Segmentation results from front angle (a) Actual Image (b) Laplacian of Gaussian (c) Laplacian (d) Gaussian (e) Prewitt (f) Sobel (g) Otsu and Mean (h) Mean and ISOdata

When combined with the Otsu method, the output of the sobel algorithm produced disappointing results. Prewitt, LOG filters, 
and Laplacian filters cannot be used in succession with any of the thresholding approaches, as illustrated in Figure 4.

This result may be explained by the fact that the filters computed gradients near zero. As a result, the photos lost nearly all of their brilliant pixels. When paired with ISOdata, the mean image filter, which operates as a low-frequency filter, yielded below average quality segmentation. The cause for this was that it was using excessive interpolation to compute the blood vessels, and as a result, the pixels became clustered. The combination of the Gaussian filter and ISOdata, as well as the sobel filter and Otsu produce equivalent results. It should be highlighted that the optimal combination was Otsu along with a mean filter with a $9 \times 9$ grid size.

\subsection{Segmentation of Optical Disc}

Optic disc is the human eye portion that passes bytes of visual stimuli to the brain neurons. It is placed in the base of the eye (Figure 5), near the center of the nose. It is the part of the eye that is harmed or swollen by glaucoma or a medical condition like papilledema. The geometric qualities like the size, area, etc. on the optical disk of the eye are used for diagnosing such medication disorders. The normal ratio of cup to disk is about $1 / 3$ or 0.3 in general (the diameter of the cup divided by the diameter of the entire nerve head or disc) [36]. There is some natural diversity here, with some people having essentially no cup (a cup to disc ratio of $1 / 10$ or 0.1 ) and others having $4 / 5$ ths or 0.8 .

Table 2: Segmentation Results of Optical Disc
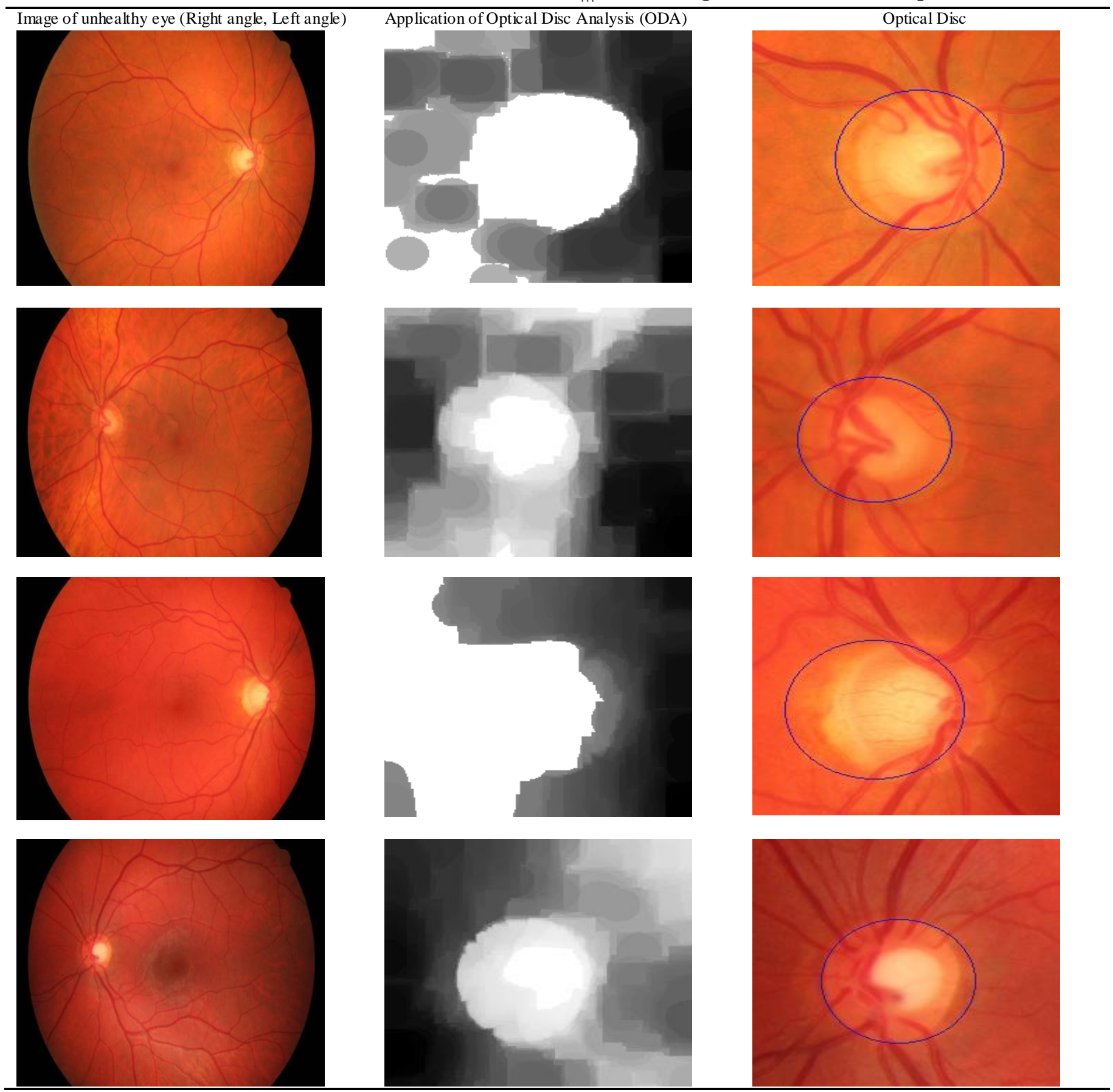
If an individual's cup/disc ratio is greater than $1 / 3$, it is thought that the cup is growing larger. Glaucoma, a medical condition, will cause the cup to expand (actually, little nerve fibers are being wiped out along the rim of the optic nerve in glaucoma). In many cases, the specialist would also examine the optic nerve's colour, as many other optic nerve diseases may cause swollen cups and the nerve to appear pale. Hence, in case detection of glaucoma, detection of the colour, texture, and geometric properties are essential. This section focuses on the segmentation of the optical disc so that accurate measurements can be taken for determining glaucoma or any other clinical investigations.

The segmentation process consists of multiple steps and is summarized as follows:

Step 1: It consists of the application of the median filter and bilateral filter on the grayscale image. The median filter helps to reduce the intensity variation between the pixels so that the image becomes smooth with medium intensity. At the same time, it helps remove salt and pepper noise in the image. The bilateral filter further smoothens the image pixels and diminishes the influence of noise further on the image. These contours in the image become more prominent. To further increase the difference between the edges, the Contrast Limited Adaptive Histogram Equalization (CLAHE) algorithm was used so that segmentation could be accessible to a higher level of contrast.

Step 2: Morphological operations such as erode and dilation were applied. In the erode operation, the boundary pixels were reduced and dilated, increasing the size of the foreground pixels. A custom function that detects the brightest pixels and most prominent object in the image was created for the mask. The segmentation process was completed using the mask created in the last step. Table 2 gives the segmentation results after the extraction of the optical disk, the geometric features of the segmented optical disk are measured and if the values of the optical disk are more than the average normal size of the healthy patient's optical disk values.

\subsection{Segmentation of Exudates}

As previously stated, diabetes patients face an elevated risk of visual loss. Eye issues can be diagnosed in conjunction with other clinical investigations and tests using diabetic retinopathy diagnosis techniques. Yellow or white lesions, also known as hard exudates $(\mathrm{EX})$, are one of the most common alterations noticed on the eye as a result of diabetes. As a result, it is evident in fundus images and is the most crucial feature to detect using a computer vision algorithm. Post-processing techniques are required to distinguish $\mathrm{EX}$ from other bright artefacts such as cotton wool spots and optical discs. This section covers how hard exudates are segmented. Table 3 gives the output after segmenting the exudates.

In conventional k-means, the outcome of the clusters and its quality depends on the initial guess given to the k-means algorithm. Since, the intention is to separate the exudates from the rest of the eye structure. It is better to give an intelligent guess to the k-means rather than giving it average values of the data. Hence, in the first step, bright objects are identified and the pixel intensity values from the both axes are given to the kmeans algorithm as initial guess.

Step 1: Find top four maximum mean values intensities of the images by sorting values from highest to lowest. The exudates have high brightness and intensity. The top four values are based on the histogram analysis which the algorithm used before choosing the starting point for execution of the loop for finding clusters.

Step 2: Initialize the cluster centres by assigning top four maximum intensity values extracted in the first step. Initialization of other routines parameters of $\mathrm{k}$ - means is done here.

Step 3: Define the constant $\mathrm{W}$, where $\mathrm{W}$ is referred to as "Width". Factor represents the typical size of the exudate cluster. If the number of elements in a cluster is greater than the width, the k-means algorithm computes another cluster until all clusters are formed. The width represents the minimum size of the exudate.

Step 4: Iterate and compute distances, update and compute new centroid till the stopping criteria get invoked.

[Histogram_means] $]=$ mean $($ histogram $([$ Image-Data $]$, bins=255))

[intial_mean0,intial_mean1,intial_mean2,intial_mean3,intial_ mean4] $=$ Sorted $(\max ($ Histogram_means $)$, asc ending $)$

Table 3: Segmentation of Exudates
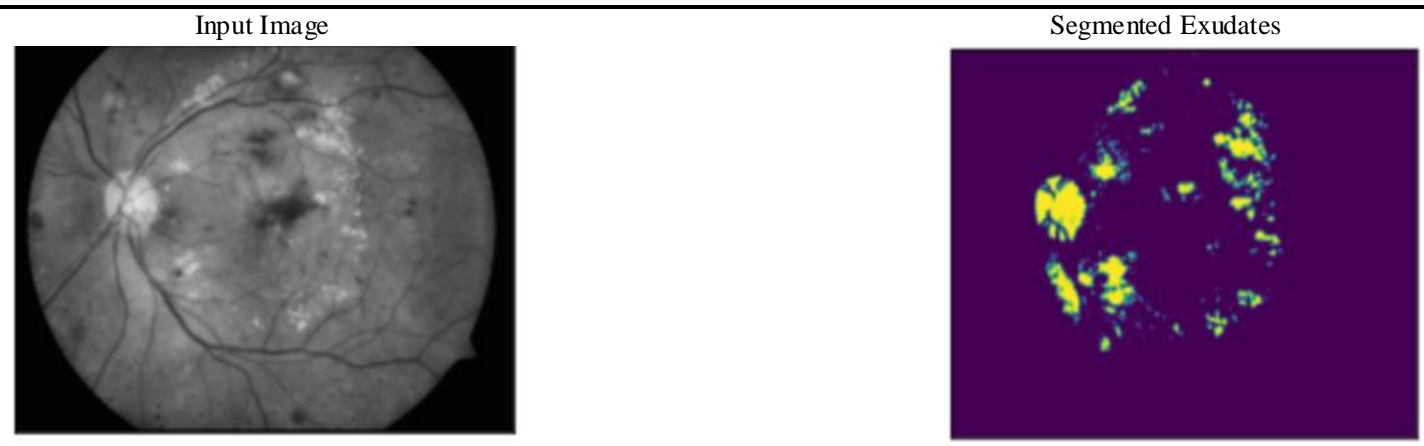
Table 4: Overall Accuracy Percentage

\begin{tabular}{|c|c|c|c|c|c|c|c|}
\hline \multirow[t]{2}{*}{ Sr. No. } & \multirow[t]{2}{*}{ Algorithm Stage } & \multirow{2}{*}{$\begin{array}{l}\text { Related Work } \\
\text { Reference }\end{array}$} & \multicolumn{3}{|c|}{ Accepted/Sample } & \multirow{2}{*}{$\begin{array}{c}\text { Average Intersection } \\
\text { over Union (IoU) } \\
\text { (Related Work) }\end{array}$} & \multirow{2}{*}{$\begin{array}{c}\text { Average Intersection } \\
\text { over Union (IoU) } \\
\text { Proposed Algorithm }\end{array}$} \\
\hline & & & 30 & $\mathbf{5 0}$ & 75 & & \\
\hline 1 & $\begin{array}{l}\text { Blood Vessel } \\
\text { Segmentation }\end{array}$ & & 20 & 23 & 39 & 0.88 & 0.99 \\
\hline 2 & $\begin{array}{l}\text { Optical Disk \& Cup } \\
\text { Analysis[38] }\end{array}$ & & 13 & 31 & 39 & 0.678 & 0.78 \\
\hline 3 & $\begin{array}{c}\text { Exudates } \\
\text { Segmentation [39] }\end{array}$ & & 15 & 39 & 67 & 0.77 & 0.98 \\
\hline
\end{tabular}

\section{RESULTS AND DISCUSSION}

This section discusses the performance of the various components of the segmentation algorithm. The segmentation algorithm's performance was evaluated at each analysis stage following a rigorous regime for evaluation. The three columns in Table 4 represent the three stages of the segmentation process. From this table, it is clear that the overall accuracy of the system is close to $91.66 \%$. The evaluation of the integrated segmentation algorithm was done in each stage. A simple random sampling method was used to check the accuracy of the segmentation process. Each item (segmented image) had equal probability to get evaluated for performance checking. Random samples of thirty, forty and seventy five were considered and with the help of IoU metric each segmented image was checked against a benchmarked set of images. The set of the benchmarked images was taken from the total population of the images with help of experts. The second observation that can be made is that at each stage with the same datasets mentioned earlier a comparative analysis was done and it was found that our algorithm overcomes the inadequacies of the previous algorithms. In the blood vessels segmentation a principle component analysis along the best convolution filter worked well and in the case of optical disk a custom function that can extract and mark the optical disk is doing fine as compared to [38]. The Exudates method is also performing well as compared to the [39], as it is using histogram based bins method to identify intensities initially to give intelligent guess to the $\mathrm{k}$-mean methods to extract exudates.

\section{CONCLUSION}

To summarize, this study describes the development of a unique segmentation method that may be used to assess the health of numerous eye parts at once. Because this algorithm can analyze multiple areas of the eyes at the same time, it has the potential to create the groundwork for the development of multi-disease detection systems. Furthermore, the nature of the segmentation technique is generic in that it may be applied to a variety of datasets while maintaining a high level of accuracy.

This study reveals that it is time-consuming and difficult to identify diseased blood vessels from normal blood arteries in the body. When evaluating the accuracy of the segmentation and then validating it, random sampling has been used in this research work at each stage. This has been done using the IoU metric. The accuracy of the integrated segmentation method as a whole is 91.66 percent. Aiming to improve the understanding of blood vessel morphology, this segmentation work seeks to identify the characteristics of healthy and pathological blood vessels in order to discriminate between the two types of vessels. Second, it assists in the segmentation of optical discs and exudates. This segmentation procedure assists medical experts in making more accurate diagnoses since the region of concern becomes more explicit in terms of evaluating the thickness, shape, and overall health of the blood arteries with which they are dealing. This research effort demonstrates that Otsu, in conjunction with the global thresholding algorithm, is the optimum method for segmenting blood vessels in the eyes from a technological standpoint. Medical imaging advancements can provide improved image resolution and enable the creation of automated algorithms that can assist in the identification and assessment of diseased blood vessels, among other benefits. Further, it is possible that machine learning techniques will be used to segment data in the future, allowing for the classification of various eye disorders and the detection of prevalent eye diseases.

\section{REFERENCES}

[1] S. M. Saleem, L. R. Pasquale, P. A. Sidoti, and J. C. Tsai, "Virtual Ophthalmology: Telemedicine in a COVID-19 Era," Am. J. Ophthalmol., vol. 216 , no. 12 , pp. 237-242, 2020.

[2] R. Sayeed, D. Gottlieb, and K. D. Mandl, "SMART Markers: collecting patient-generated health data as a standardized property of health information technology," npj Digit. Med., vol. 3, no. 1, 2020, doi: 10.1038/s41746-020-0218-6.

[3] M. Ponnibala, E. B. Priyanka, and S. Thangavel, "Proliferative Diabetic Retinopathy Diagnostic Investigation Using Retinal Blood Vessels Mining Technique," Sens. Imaging, vol. 22, no. 1, 2021, doi: 10.1007/s11220-02100331-9

[4] K. Mittal and V. M. A. Rajam, "Computerized retinal image analysis - a survey," Multimed. Tools Appl., vol. 79, no. 31-32, pp. 22389-22421, 2020, doi: 10.1007/s11042-020-09041-y.

[5] F. Abdullah et al., "A Review on Glaucoma Disease Detection Using Computerized Techniques," IEEE Access, vol. 9. pp. 37311-37333, 2021, doi: 10.1109/ACCESS.2021.3061451.

[6] A. M. Syed, M. U. Akbar, and J. Fatima, "An overview of oct techniques for detection of ophthalmic syndromes," in EAI/Springer Innovations in 
Communication and Computing, 2019, pp. 109-116.

[7] M. H. Sarhan et al., "Machine Learning Techniques for Ophthalmic Data Processing: A Review," IEEE J. Biomed. Heal. Informatics, vol. 24, no. 12, pp. 3338-3350, 2020, doi: 10.1109/JBHI.2020.3012134.

[8] E. Y. K. Ng, U. R. Acharya, A. Campilho, and J. S. Suri, Image analysis and modeling in ophthalmology. 2014.

[9] R. Bernardes, P. Serranho, and C. Lobo, "Digital ocular fundus imaging: A review," Ophthalmologica, vol. 226, no.4. 161-181,

$$
\text { 2011, doi: 10.1159/000329597. }
$$

[10] G. Lim, V. Bellemo, Y. Xie, X. Q. Lee, M. Y. T. Yip, and D. S. W. Ting, "Different fundus imaging modalities and technical factors in AI screening for diabetic retinopathy: a review," Eye Vis., 2020, doi: 10.1186/s40662-020-00182-7.

[11] S. Jeon, Y. Liu, J. P. O. Li, D. Webster, L. Peng, and D. Ting, "AI papers in ophthalmology made simple," Eye (Basingstoke), vol. 34, no. 11. pp. 1947-1949, 2020, doi: 10.1038/s41433-020-0929-6.

[12] D. Lokuarachchi, L. Muthumal, K. Gunarathna, and T. D. Gamage, "Detection of Red Lesions in Retinal Images Using Image Processing and Machine Learning Techniques," in MERCon 2019 - Proceedings, 5th International Multidisciplinary Moratuwa Engineering Research Conference, 2019, pp. 550-555, doi: 10.1109/MERCon.2019.8818794.

[13] S. Long, J. Chen, A. Hu, H. Liu, Z. Chen, and D. Zheng, "Microaneurysms detection in color fundus images using machine learning based on directional local contrast," doi: 10.1186/s12938-02000766-3.

[14] D. S. Sisodia, S. Nair, and P. Khobragade, "Diabetic retinal fundus images: Preprocessing and feature extraction for early detection of Diabetic Retinopathy," Biomed. Pharmacol. J., 2017, doi: $10.13005 / \mathrm{bpj} / 1148$.

[15] S. S. Johnson, J. K. Wang, M. S. Islam, M. J. Thurtell, R. H. Kardon, and M. K. Garvin, "Local Estimation of the Degree of Optic Disc Swelling from Color Fundus Photography," in Lecture Notes in Computer Science (including subseries Lecture Notes in Artificial Intelligence and Lecture Notes in Bioinformatics), 2018, vol. 11039 LNCS, pp. 277-284, doi: 10.1007/978-3-030-00949-6_33.

[16] S. Morales, V. Naranjo, J. Angulo, A. G. Legaz-Aparicio, and R. VerdúMonedero, "Retinal network characterization through fundus image processing: Significant point identification on vessel centerline," Signal Process. Image Commun., vol. 59, 2017, doi: 10.1016/j.image.2017.03.013.

[17] T. A. Soomro, A. M. Bughio, S. H. Siyal, A. A. Panwar, and N. Nizamani, "A Wide-Ranging Review on Diabetic Retinotherapy," Quaid-e-Awam Univ. Res. J. Eng. Sci. Technol., vol. 18, no. 02, 2020, doi: 10.52584/qrj.1802.25.

[18] P. Powar and P. C. R. Jadhav, "Comparative Study of Classifiers for Diagnosis of Microaneurysm," 2016.

[19] C. Méjécase, S. Malka, Z. Guan, A. Slater, G. Arno, and M. Moosajee, "Practical guide to genetic screening for inherited eye diseases," Ther. Adv. Ophthalmol., vol. 12, 2020, doi: 10.1177/2515841420954592.

[20] T. Ratanapakorn, A. Daengphoonphol, N. Eua-Anant, and Y. Yospaiboon, "Digital image processing software for diagnosing diabetic retinopathy from fundus photograph," Clin. Ophthalmol., vol. 13, pp. 641-668, 2019, doi: 10.2147/OPTH.S195617.

[21] B. Bataineh and K. H. Almotairi, "Enhancement Method for Color Retinal Fundus Images Based on Structural Details and Illumination Improvements," Arab. J. Sci. Eng., 2021, doi: 10.1007/s13369-02105429-6.

[22] K. Kipli et al., "A Review on the Extraction of Quantitative Retinal Microvascular Image Feature," Computational and Mathematical Methods in Medicine. 2018, doi: 10.1155/2018/4019538.
[23] Y. Elloumi, M. Akil, and N. Kehtarnavaz, "A Computationally Efficient Retina Detection and Enhancement Image Processing Pipeline for Smartphone-Captured Fundus Images A Computationally Efficient Retina Detection and Enhancement Image Processing Pipeline for Smartphone-Captured Fundus," Images. J. Multimed. Inf. Syst., vol. 0, no. $0, \quad$ pp. 1-10, 2018, [Online]. Available: http://dx.doi.org/00.0000/JMIS.0000.00.0.000.

[24] Z. Shen, H. Fu, J. Shen, and L. Shao, "Modeling and Enhancing LowQuality Retinal Fundus Images," IEEE Trans. Med. Imaging, vol. 40, no. 3, pp. 996-1006, 2021, doi: 10.1109/TMI.2020.3043495.

[25] G. J. Anitha and K. G. Maria, "Detecting Hard Exudates in Retinal Fundus Images Using Convolutinal Neural Networks," 2018, doi: 10.1109/ICCTCT.2018.8551079.

[26] C. Bhardwaj, S. Jain, and M. Sood, "Hierarchical severity grade classification of non-proliferative diabetic retinopathy," J. Ambient Intell. Humaniz. Comput., 2020, doi: 10.1007/s12652-020-02426-9.

[27] S. S. Chowhan, R. S. Deore, and S. A. Naik, "Retinal Vessel Segmentation of Non-Proliferative Diabetic Retinopathy," Int. J. Appl. Evol. Comput., 2018, doi: 10.4018/ijaec.2019010102.

[28] M. S. Mabrouk, N. H. Solouma, and Y. M. Kadah, "Survey of Retinal Image Segmentation and Registration," GVIP J., vol. 6, no. 2, p. 1, 2006

[29] M. M. Fraz et al., "Blood vessel segmentation methodologies in retinal images - A survey," Comput. Methods Programs Biomed., vol. 108, no. 1, pp. 407-433, 2012, doi: 10.1016/j.cmpb.2012.03.009.

[30] C. Zhu et al., "A survey ofretinal vessel segmentation in fundus images," Jisuanji Fuzhu Sheji Yu Tuxingxue Xuebao/Journal Comput. Des. Comput. Graph, vol. 27, no. 11, pp. 2046-2057, 2015.

[31] P. Powar and C. R., "A Survey of Microaneurysms Detection using Segmentation Techniques in Fundus Images," Int. J. Comput. Appl., vol. 135, no. 1, pp. 32-34, 2016, doi: 10.5120/ijca2016908311.

[32] A. Raj, A. K. Tiwari, and M. G. Martini, "Fundus image quality assessment: Survey, challenges, and future scope," IET Image Process., vol. 13, no. 8, pp. 1211-1224, 2019, doi: 10.1049/iet- ipr.2018.6212.

[33] S. Joshi and P. T. Karule, "Mathematical morphology for microaneurysm detection in fundus images," Eur. J. Ophthalmol., vol. 30, no. 5, pp. 1135-1142, 2020, doi: 10.1177/1120672119843021.

[34] R. K. Singh and R. Gorantla, "DMenet: Diabetic Macular Edema diagnosis using Hierarchical Ensemble of CNNs," PLoS One, 2020, doi: 10.1371/journal.pone.0220677.

[35] K. Lin, Li; Li, Meng; Huang, Yijin; Cheng, Pujin; Xia, Honghui; Wang, "The SUSTech-SYSU dataset for automated exudate detection and diabetic retinopathy grading." 2020.

[36] J. B. Jonas, W. M. Budde, and S. Panda-Jonas, "Ophthalmoscopic evaluation of the optic nerve head," Survey of Ophthalmology, vol. 43, no. 4. Elsevier Inc., pp. 293-320, 1999, doi: 10.1016/S00396257(98)00049-6.

[37] https://learning-center.homesciencetools.com/article/eye-and-vision/

[38] https://medium.com/@gautamkumarjaiswal/retina-blood-vesselsegmentation- using-matlab-ce 5 cfd 1 fa974

[39] Bagadam David (2021). Localization of Hard Exudates in RetinalFundus Images (https://www.mathworks.com/matlabcentral/fileexchange/6954 2-localization-of-hard-exudates-in-retinal-fundus-images), MATLAB Central File Exchange. Retrieved December 14, 2021.

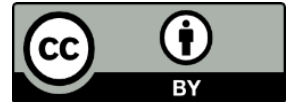

(C) 2021 by the Parul Datta, Prasenjit Das and Abhishek Kumar. Submitted for possible open access publication under the terms and conditions of the Creative Commons Attribution (CC BY) license (http://creativecommons.org/licenses/by/4.0/). 